\title{
Anaplastic Lymphoma Kinase (ALK) and p53 Are Potentially Useful Markers to Distinguish Inflammatory Myofibroblastic Tumor
}

\author{
Shinji Kurosaka ${ }^{1}$, Kazumasa Matsumoto ${ }^{1}$, Akira Irie ${ }^{1}$, Takahiro Hirayama ${ }^{1}$, Morihiro Nishi ${ }^{1}$, \\ Tetsuo Fujita ${ }^{1}$, Takefumi Satoh ${ }^{1}$, Yuichi Sato ${ }^{2}$, Masatsugu Iwamura ${ }^{1}$, Kazunari Yoshida ${ }^{1}$ \\ ${ }^{1}$ Department of Urology, School of Medicine, Kitasato University, Tokyo, Japan \\ ${ }^{2}$ Department of Molecular Diagnostics, School of Allied Health Sciences, Kitasato University, Tokyo, Japan \\ Email: kurosaka969696@yahoo.co.jp
}

Received December 29, 2012; revised January 31, 2013; accepted February 10, 2013

Copyright (C) 2013 Shinji Kurosaka et al. This is an open access article distributed under the Creative Commons Attribution License, which permits unrestricted use, distribution, and reproduction in any medium, provided the original work is properly cited.

\begin{abstract}
Aims: Inflammatory myofibroblastic tumor (IMT) of the urinary bladder is a clinically and histologically uncommon benign tumor that can be easily mistaken for a malignant neoplasm. We sought to determine whether immunohistochemical staining would be evaluated IMT of the urinary bladder. We have also shown the literatures that imminohistochemical staining of IMT was investigated to distinguish malignant lesions using PubMed data base. Methods: Immunohistochemical staining, including anaplastic lymphoma kinase (ALK), p53, cytokeratin, vimentin, desmin, alpha-smooth muscle actin, myoglobin, smooth muscle myosin and S100, was carried out on serial sections from archival specimens of three patients who underwent transurethral resection and partial cystectomy. Results: Immunohistchemical staining in all patients was positive for ALK and weak positive for p53 protein. In the literatures, positive rates of ALK and p53 in the IMT of the urinary bladder were $60.9 \%$ and $53.1 \%$, respectively. Sarcoma and carcinosarcoma were shown in the pathological specimens with negative ALK and strongly positive p53 in the same data base. Conclusions: Both ALK and p53 were potentially useful protein markers to distinguish between IMT and sarcoma. However, this study was small sample size. Further study was warranted an investigation of the availability of these proteins in IMT.
\end{abstract}

Keywords: Inflammatory Myofibroblastic Tumor; Immunohistochemistry; Bladder Cancer; Anaplastic Lymphoma Kinase; p53

\section{Introduction}

Inflammatory myofibroblastic tumor (IMT) consists of myofibroblastic spindle cells associated with lymphoplasmocytic and eosinophilic inflammatory infiltrate. IMT of the urinary bladder is a rare benign lesion that can be easily mistaken for a malignant neoplasm, because IMT is rapid growth during a short time of period.

Recently, anaplastic lymphoma kinase (ALK) gene chromosomal rearrangement or ALK protein expression has been reported in some cases of IMT [1,2]. Over expression or activation of ALK is known to be involved in the genesis of a subset of anaplastic large cell lymphomas and has helped in redefining the entity [3].

We sought to determine whether immunohistochemical staining would be evaluated IMT of the urinary bladder. In addition, we have also shown the literatures that were investigated imminohistochemical staining of IMT to distinguish the malignant lesions using PubMed data base.

\section{Patients and Methods}

We studied three patients with IMT of the urinary bladder (Table 1). There were two males and one female: mean age was 29.7 years (range 21 - 44). Histopathologic characteristics were confirmed by blinded review of the original pathology slides (Y.S.). Two patents were treated with TUR and one patient was performed partial cystectomy. No patient was treated preoperatively with either radiation or systemic chemotherapy. We collected formalin-fixed, paraffin-embedded blocks representing the most appearance areas of each tumor. We performed ALK, p53, cytokeratin, vimentin, desmin, alpha-smooth 
Table 1. Characteristics of the inflammatory myofibroblastic tumor in three patients.

\begin{tabular}{cccc}
\hline & Case1 & Case2 & Case3 \\
\hline Age & 21 & 44 & 24 \\
Gender & Male & Female & Male \\
Chief complaint & Hematuria & Hematuria & Perineal pain \\
Location & Dome & Anterior wall & Dome \\
Tumor size & $5 \mathrm{~cm}$ & $3 \mathrm{~cm}$ & $5 \mathrm{~cm}$ \\
Tumor form & Non-papillary & Non-papillary & Non-papillary \\
& Pediculate & Pediculate & Sessile \\
& $\begin{array}{c}\text { Invasive } \\
\text { Transurethral } \\
\text { resection }\end{array}$ & $\begin{array}{c}\text { Non-invasive } \\
\text { Transurethral } \\
\text { resection }\end{array}$ & $\begin{array}{c}\text { Invasive } \\
\text { Partial } \\
\text { cystectomy }\end{array}$ \\
\hline
\end{tabular}

muscle actin ( $\alpha$-SMA), myoglobin, smooth muscle myosin (SMM) and S100 immunohistochemical staining (Table 2) as previously described [4]. All markers were placed in one of two categories, altered or normal, by at least two investigators, who were blinded to clinical outcome.

When we researched ALK staining in IMT using the Pub Med database, there are 11 literatures. There are 84 cases of ALK staining in IMT. On the other hand, we checked p53 staining in IMT using same database. There are 46 cases of p53 staining in IMT (Table 3).

\section{Results}

All patients were revealed solid and sessile tumor in the bladder wall. Histologically, there was the proliferation of plump spindle fibroblasts without abnormal mitosis in inflammatory background. (Figure 1) ALK (Figure 2) and vimentin staining was positive in all three patients (Table 1). p53 expressions were weak but positive in all three patients. (Figure 3) Two of three patients had the positive intensity of desmin and $\alpha$-SMA. One of three patients had positive staining in cytokeratin. Immunohistochemical staining of myoglobin, SMM, S-100 protein was negative in all three patients.

The PubMed database reported 11 literatures shown ALK staining in IMT. Investigation of ALK staining was demonstrated 87 patients including our three patients (mean age, 43.3 years) in IMT. ALK was positive in 53 patients $(60.9 \%)$. Utilizing the same data base, p53 staining in IMT of the urinary bladder was reported in three literatures $[2,5]$. According to them, immunohistochemical staining of $\mathrm{p} 53$ was shown 49 patients. p53 was positive in 26 patients $(53.1 \%)$.

\section{Discussion}

The first case of IMT was reported by Roth in 1980 [6].
Table 2. Immunohistochemical reactivities in inflammatory myofibroblastic tumor.

\begin{tabular}{cccc}
\hline Antigen & Case1 & Case2 & Case3 \\
\hline AE1 & - & - & + \\
Vimentin & + & + & $+/-$ \\
Desmin & + & + & - \\
HHF-35 & - & + & - \\
$\alpha$-SMA & + & + & - \\
myoblobin & - & - & - \\
SMM & - & - & - \\
S-100 & - & - & - \\
ALK & + & + & + \\
p53 & + & + & + \\
\hline
\end{tabular}

$\alpha$-SMA $=\alpha$-smooth muscle actin; SMM $=$ smooth muscle myosin; $\mathrm{ALK}=$ anaplastic lymphoma kinase.

Table 3. Reactivity of ALK in 87 patients of Inflammatory myofibroblastic tumor.

\begin{tabular}{cccc}
\hline Author & Cases & ALK expression & Mean age \\
\hline Allory Y. & 1 & $1 / 1$ & 78 \\
Chan J.K. & 6 & $2 / 6$ & 54.3 \\
Debiec-Rycher M. & 1 & $1 / 1$ & 46 \\
Freeman A. & 9 & $8 / 9$ & 36.5 \\
Harik L.R. & 26 & $12 / 26$ & 47 \\
Heney N.M. & 1 & $1 / 1$ & 33 \\
Hirsch M.S. & 21 & $10 / 21$ & 40.6 \\
Takeshita H. & 1 & $1 / 1$ & 52 \\
Tsuzuki T. & 16 & $12 / 16$ & 39.2 \\
Yamashita R. & 1 & $1 / 1$ & 39 \\
Hayashi T. & 1 & $1 / 1$ & 72 \\
Present study & 3 & $3 / 3$ & 29.7 \\
Total & 87 & $53 / 87$ & 43.3 \\
\hline
\end{tabular}

IMT was known as many different names, including inflammatory pseudotumor, inflammatory pseudosarcomatous fibromyxoid tumor, pseudosarcomatous myofibroblastic tumor and so on. However, these names are unified as inflammatory myofibroblastic tumor (IMT) [7]. While IMT possibly occurred at any anatomical site, lung lesions are mostly reported. IMT detected in the urinary bladder is a rare entity. IMT of the urinary bladder is an 


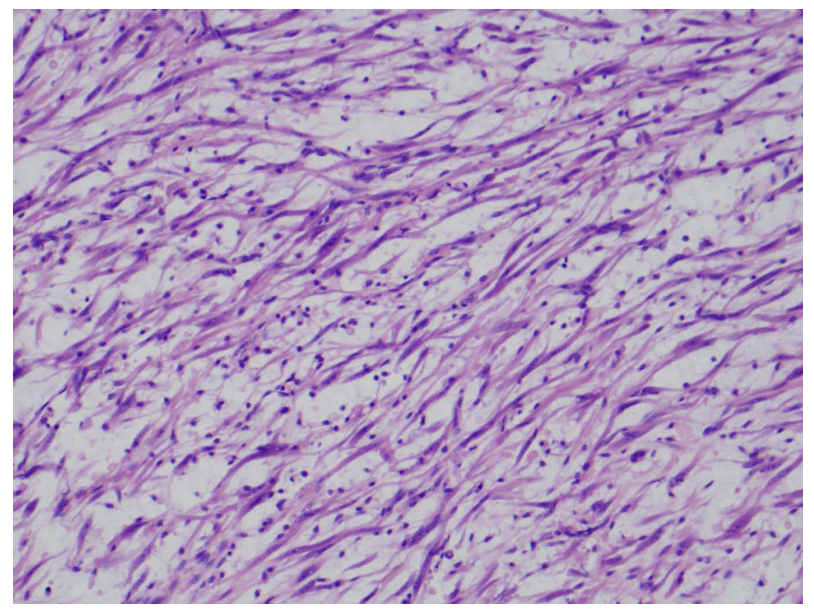

Figure 1. Histopathological examination reveals proliferation of spindle cells and inflammatory cells (HE stain).

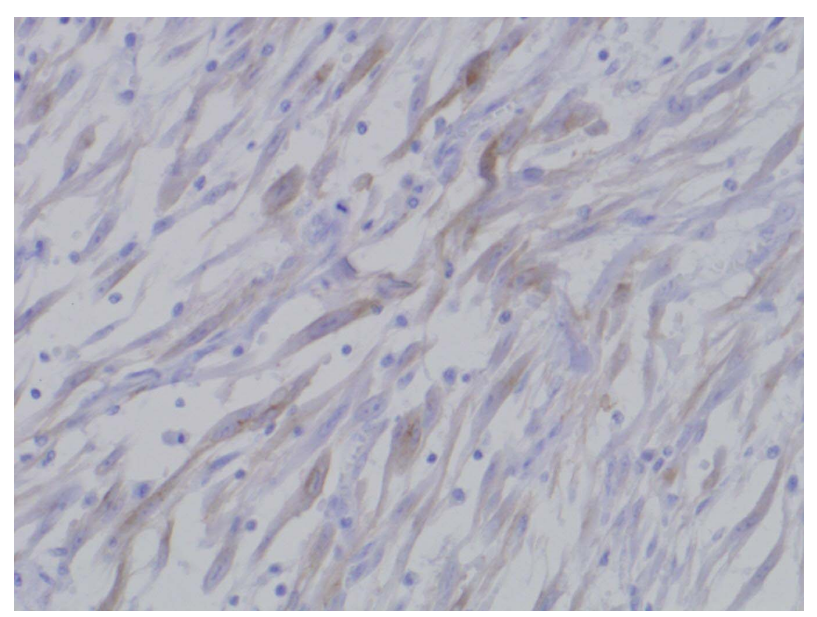

Figure 2. Inflammatory myofibroblastic tumor showed positive immunoreactivity of ALK.

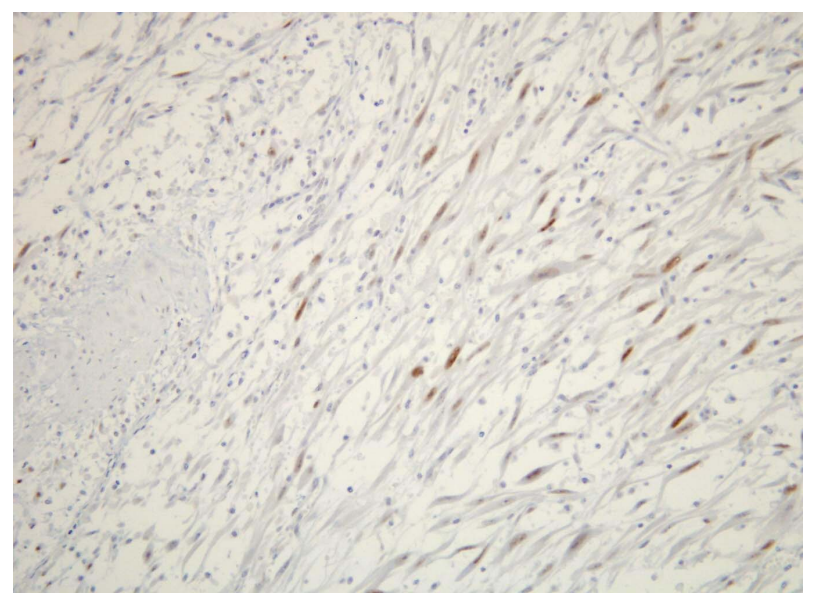

Figure 3. p53 protein was weak positive in inflammatory myofibroblastic tumor.

uncommon mesenchymal neoplasm composed of spindle cells with an accompanying lymphoplasmacytic infiltrate.
Because of its cytologic features and infiltrative nature, it may be often difficult to distinguish from histologically sarcomatous increases such as sarcomatoid urothelial carcinoma, leiomyosarcoma, and rhabdmyosarcoma [8,9]. Immunohistochemistry may aid in the differential diagnosis of spindle cell lesions, including bladder. Table 4 showed the various immunohistochemical profiles. Sarcomatous lesions demonstrated that most of the immunohistochemical staining has been shown weak or moderate expression compared to IMT. These results were strengthened this study using immunohistochemical profiles in terms of distinguishing from sarcomatous lesions to IMT.

ALK was first identified in a portion of anaplastic large cell lymphoma [3], and ALK reactivity correlates with local recurrence and muscle invasion in cancerous lesions. Recently, some IMTs of the urinary bladder have showed clonal abnormalities involving the ALK gene, and immunoreactivity of ALK protein [1,2]. On the other hand, the expression of ALK is not detected in the majority of cases of sarcomatoid carcinomas, leiomyosarcomas, or rhabdmyosarcomas of the bladder [2]. Freeman et al. demonstrated that ALK immunoreactivity was positive in eight of nine patients $(89 \%)$ of the vesical IMT, however patients with either sarcomatooid carcinoma, leiomyosarcoma, rhabdomyosarcoma, or neurofibromas were all negative in terms of ALK expression [10]. In this study utilizing other previous reports, ALK were positive in $60.9 \%$ of IMT patients. We believe utilization of ALK immunoreactivity in IMT of the urinary bladder was one of the options to distinguish between IMT and sarcomatoid lesions.

p53 protein is tumor suppressor gene, and it is often measured as tumor marker in a malignant potency. According to the reports of Iczkoski et al. [11] and Tsuzuki et al. [2] p53 protein of IMT in the urinary bladder has been shown weak staining. In addition, sarcoma showed a strong staining of p53 expression. They reported that p53 protein was one of available tumor markers for IMT. In our three patients, p53 protein was weak positive. Immunoreactivity of p53 may assist to check biological aggressiveness of IMT. Interestingly, Hussong et al. [5] reported two patients $(8 \%)$ revealed strong p53 expression in vesical IMT. p53 alteration in IMT would have a possibility of malignant potential and meditated on a closed follow-up carefully.

Limitation of this study is a small sample size, however, accumulation of previous reports including our three cases would show one of the clues to distinguish between inflammatory benign lesion and malignancy using immunohistochemistry of ALK and p53. IMT itself is a rare tumor, in addition to this, IMT of the urinary bladder is a much rarer tumor. Multi-institutional col- 
Table 4. Immunohistochemical expressions in IMT and sarcomatous lesions.

\begin{tabular}{|c|c|c|c|c|c|c|c|c|c|}
\hline & AE1 & Des. & SMA & S-100 & CK & Calp. & Cald. & Мyo. & ALK \\
\hline \multirow[t]{2}{*}{ IMT } & $+/-$ & $+/-$ & $+/-$ & - & $-1+$ & $+/-$ & $+/-$ & - & $+/-$ \\
\hline & $\begin{array}{c}30 \%- \\
80 \%\end{array}$ & $0-80 \%$ & $<90 \%$ & & $\begin{array}{c}20 \%- \\
40 \%\end{array}$ & & & & $\begin{array}{c}33 \% \\
75 \%\end{array}$ \\
\hline \multirow[t]{2}{*}{ Leiomyo-sarcoma } & $-/+$ & + & + & - & $-/+$ & + & + & - & - \\
\hline & $0-10 \%$ & & & & $30 \%<$ & & & & \\
\hline \multirow[t]{2}{*}{ Rhabdomyo-sarcoma } & $-/+$ & + & $-/+$ & - & $-1+$ & - & - & + & $-/+$ \\
\hline & $0-10 \%$ & & & & & & & & $20 \%$ \\
\hline Sarcomatoid urohelial & $+/-$ & $-1+$ & $-/+$ & - & + & - & - & - & - \\
\hline Sarcoma & $<75 \%$ & $<25 \%$ & $<25 \%$ & & & & & & \\
\hline
\end{tabular}

CK: Cytokeratin; SMA: Smooth muscle actin; Des.: Desmin; Calp.: Calponin; Cald.: Caldesmon; Myo.: Myogenin; ALK: Anaplastic lymphoma kinase.

laboration will lead to resolve clinical problem and biological behavior, and finally contribute to the patients who suffered from such a rare tumor.

\section{REFERENCES}

[1] J. K. Chan, W. Cheuk and M. Shimizu, "Anaplastic Lymphoma Kinase Expression in Inflammatory Pseudotumors," American Journal of Surgical Pathology, Vol. 25, No. 6, 2001, pp. 761-768. doi:10.1097/00000478-200106000-00007

[2] T. Tsuzuki, C. Magi-Galluzzi and J. I. Epstein, "ALK-1 Expression in Inflammatory Myofibroblastic Tumor of the Urinary Bladder," American Journal of Surgical Pathology, Vol. 28, No. 12, 2004, pp. 1609-1614. doi:10.1097/00000478-200412000-00009

[3] S. W. Morris, M. N. Kirstein, M. B. Valentine, K. G. Dittmer, D. N. Shapiro, D. L. Saltman and A. T. Look, "Fusion of a Kinase Gene, ALK, to a Nucleolar Protein Gene, NPM, in Non-Hodgkin's Lymphoma," Science Magazine, Vol. 263, No. 5151, 1994, pp. 1281-1284. doi:10.1126/science. 8122112

[4] C. M. Coffin, L. P. Dehner, J. M. Meis-Kindblom and S. Diagn, "Inflammatory Myofibroblastic Tumor, Inflammatory Fibrosarcoma, and Related Lesions: An Historical Review with Differential Diagnostic Considerations," Seminars in Diagnostic Pathology, Vol. 15, No. 2, 1998, pp. 102-110.

[5] J. W. Hussong, M. Brown, S. L. Perkins, L. P. Dehner and C. M. Coffin, "Comparison of DNA Ploidy, Histologic, and Immunohistochemical Findings with Clinical Outcome in Inflammatory Myofibroblastic Tumors," Modern Pathology, Vol. 12, No. 3, 1999, pp. 279-286.
[6] J. A. Roth, "Reactive Pseudosarcomatous Response in Urinary Bladder," Urology, Vol. 16, No. 6, 1980, pp. 635-637. doi:10.1016/0090-4295(80)90578-6

[7] M. Hisaoka, S. Shimajiri, Y. Matsuki, J. M. Meis-Kindblom, L. G. Kindblom, X. Q. Li, J. Wang and H. Hashimoto, "Inflammatory Myofibroblastic Tumor with Predominant Anaplastic Lymphoma Kinase-Positive Cells Lacking a Myofibroblastic Phenotype," Pathology International, Vol. 53, No. 6, 2003, pp. 376-381. doi:10.1046/j.1440-1827.2003.01484.X

[8] K. W. Chan, K. L. Chan and K. Y. Lam, "Inflammatory Pseudotumor of Epididymis and Epstein-Barr Virus: A Study of Two Cases," Pathology, Vol. 29, No. 1, 1997, pp. 100-101.doi:10.1080/00313029700169664

[9] J. M. Meis-Kindblom, C. Kjellstrom and L. G. Kindblom, "Inflammatory Fibrosarcoma: Update, Reappraisal, and Perspective on Its Place in the Spectrum of Inflammatory Myofibroblastic Tumors," Seminars in Diagnostic Pathology, Vol. 15, No. 2, 1998, pp. 133-143.

[10] A. Freeman, N. Geddes, P. Munson, J. Joseph, P. Ramani, A. Sandison, C. Fisher and M. C. Parkinson, "Anaplastic Lymphoma Kinase (ALK 1) Staining and Molecular Analysis in Inflammatory Myofibroblastic Tumours of the Bladder: A Preliminary Clinicopathological Study of Nine Cases and Review of the Literature," Modern Pathology, Vol. 17, No. 7, 2004, pp. 765-771. doi: $10.1038 /$ modpathol.3800078

[11] K. A. Iczkowski, J. H. Shanks, V. Gadaleanu, L. Cheng, E. C. Jones, R. Neumann, A. G. Nascimento and D. G. Bostwick, "Inflammatory Pseudotumor and Sarcoma of Urinary Bladder: Differential Diagnosis and Outcome in Thirty-Eight Spindle Cell Neoplasms," Modern Pathology, Vol. 14, No. 10, 2001, pp. 1043-1051. doi:10.1038/modpathol.3880434 